marital status (married or not) were the exposures. The outcomes were hypertension, diabetes, hypercholesterolaemia, smoking, sedentariness, abdominal obesity, diet, alcohol consumption and depression. Subjects were grouped as $0-1,2-3$ or $\geq 4$ RF. Binomial and multinomial logistic regressions were used to estimate ageadjusted ORs.

Results Among women, both lower education and occupation were associated with hypertension, diabetes, sedentariness, abdominal obesity, poor diet, excessive alcohol intake and depression. Smoking was less prevalent among married women, with lower education and occupation. Among men, lower education and occupation were associated with sedentariness and excessive alcohol intake. Diabetes was more prevalent among less educated men. Being married increased the odds of hypertension in women but decreased the odds of smoking and poor diet in men. The effects of education and occupation were stronger in subjects with $\geq 4 \mathrm{RF}$ than in those with 2-3 RF (reference: 0-1 RF).

Conclusion Overall, lower social standing is associated with adverse cardiovascular risk profiles in a dose-response manner. Genderspecific patterns emerge between education, occupation and marital status across established cardiovascular risk factors, contributing to clarify mechanisms linking socioeconomic position to cardiovascular health.

\section{P2-12 RECURRENT DENTURE RELATED SORES AND ORAL CANCER}

doi:10.1136/jech.2011.142976h.49

${ }^{1} \mathrm{~L}$ D B Rotundo, ${ }^{2} \mathrm{~T} N$ Toporcov, ${ }^{1} \mathrm{M}$ G Haye Biazevic, ${ }^{3,2} \mathrm{M}$ B de Carvalho, ${ }^{2} J$ L Ferreira Antunes. ${ }^{1}$ Faculdade de Odontologia, Universidade de Sao Paulo, Sao Paulo, Sao Paulo, Brazil; ' 2 Faculdade de Saude Publica, Universidade de Sao Paulo, Sao Paulo, Sao Paulo, Brazil; ${ }^{3}$ Hospital Heliopolis, Sao Paulo, Sao Paulo, Brazil

Background The potential contribution of denture-related sores to oral carcinogenesis still fuels controversies. This study assessed the association between recurrent denture-related sores and squamous cell carcinoma in anatomic sites of the mouth that are specifically at risk of sores by ill-fitting dentures.

Methods We conducted a hospital-based case-control study comprising 71 histologically-confirmed cases of squamous cell carcinoma in anatomic sites of the mouth that were specifically at risk of developing sores due to ill-fitting dentures, who were attended at two hospitals in São Paulo, Brazil; and 240 controls without cancer, recruited from outpatient units of the same hospitals. Associations were assessed by multivariate logistic regression conditioned on socio-demographic (family income) and behavioural characteristics (tobacco smoking, alcohol drinking and dietary patterns)

Results The association between ill-fitting dentures and mouth cancer was statistically significant in the multivariate model, which adjustment for socio-demographic and behavioural characteristics: $\mathrm{OR}=3.98$ (95\% CI 1.06 to 14.96). The specific assessment of association between tumours in the lower jaw and sores by mandibular dentures confirmed this result ( $O R=6.39 ; 95 \%$ CI 1.49 to 29.52).

Conclusions These results reinforce the hypothesis that an appropriate application and monitoring of dental prosthesis represent a non-negligible scope for cancer prevention.

\section{P2-13 FAMILY HISTORY OF CANCER: THE ROLE OF SMOKING STATUS OF AFFECTED RELATIVES}

doi:10.1136/jech.2011.142976h.50

${ }^{1} \mathrm{~T} N$ Toporcov, ${ }^{1} \mathrm{~J}$ L Ferreira Antunes, ${ }^{*} \mathrm{M}$ B de Carvalho, ${ }^{6} \mathrm{D}$ L Figueiredo, ${ }^{3} \mathrm{~J}$ F GóisFilho, ${ }^{5} \mathrm{P}$ Michaluart-Júnior, ${ }^{4} \mathrm{E} H$ Tajara, ${ }^{1} \mathrm{Head}$ and Neck Genome Project GENCAPO, ${ }^{1}$ V Wünsch-Filho. ${ }^{1}$ Faculdade de Saúde Pública, Universidade de São Paulo, São Paulo,
São Paulo, Brazil; ${ }^{2}$ Hospital Heliopolis, São Paulo, São Paulo, Brazil; ${ }^{3}$ nnstituto do Câncer Arnaldo Vieira de Carvalho, São Paulo, São Paulo, Brazil; ${ }^{4}$ Faculdade de Medicina de Ribeirão Preto, São Paulo, São Paulo, Brazil; ${ }^{5}$ Hospital das Clínicas da Faculdade de Medicina, Universidade de São Paulo, São Paulo, São Paulo, Brazil; ${ }^{6}$ Hospital das Clínicas de Ribeirão Preto, Universidade de São Paulo, São Paulo, São Paulo, Brazil

Tobacco smoking is a major risk factor for head and neck cancer (HNC). Family history of cancer is also associated with HNC. This association lies in the difficulty in balancing the influence of hereditary vs shared environmental factors. We aimed to assess the role of smoking status of affected relatives on the risk of HNC. This study comprised 567 patients with HNC and 328 controls without cancer admitted in five centers in São Paulo State, Brazil, from 2005 to 2009; all of them had at least one parent or sibling with cancer of any site reported. Multivariate unconditional logistic regression was used to assess the effect of tobacco smoking in affected relatives on the risk of HNC. ORs were adjusted on socioeconomic status, tobacco smoking, alcohol drinking, age, sex and the number of relatives that experienced cancer. Having one or more smoker affected relative was associated with a higher risk of HNC $(\mathrm{OR}=2.39 ; 95 \%$ CI 1.69 to 3.37). This association remained high when stratification by cancer subsites was performed: oral cavity (2.11; 1.41 to 3.17 ), oropharynx (2.84; 1.54 to 5.23$)$, larynx (4.05; 2.36 to 6.93). In conclusion, cancer affected relatives of HNC cases are more exposed to tobacco smoking than cancer affected relatives of controls. This finding suggests that lifestyle factors of parents or siblings with cancer history may play a role on the association between family history of cancer and HNC.

\section{P2-14 STUDY OF THE QUALITY OF LIFE IN PATIENTS AFTER URINARY STONE FRAGMENTATION}

doi:10.1136/jech.2011.142976h.51

1,2M Arafa, * ${ }^{1} \mathrm{D}$ Rabah. ${ }^{1}$ King Saud University, Riyadh, Saudi Arabia; ${ }^{2}$ Princess Al Johara Al Ibrahim Center for Cancer Research, Riyadh, Saudi Arabia

This study was designed to evaluate the health-related quality of life (HRQoL) of patients who had undergone lithotripsy for treatment of urinary stones and to identify factors that significantly affect their HRQOL.

Methods A comparative cross-sectional study was performed at the main university hospital in Riyadh, Saudi Arabia. All patients admitted to the urology service for urinary stones fragmentation during a 9-month period were included in the study. An observation period of 3-15 months following the last treatment was allowed before patients completed the QOL questionnaire. Information on socio-demographic, medical characteristics, number and type of lithotripsies were collected. The Medical Outcome Study ShortForm 36-item survey (SF-36) was used to assess HRQoL. For comparison, the HRQoL in an equal number of healthy individuals was investigated; multivariate analysis of variance was used for comparisons between groups.

Results Compared with healthy subjects, lithotripsy patients had significantly higher mean scores in the different subscales of the SF36 questionnaire such as physical functioning, vitality, role-physical, role-emotional and mental health, indicating a better HROOL. Compared with patients who underwent ureteroscopic or extracorporeal shock-wave lithotripsies, those who underwent percutaneous lithotripsy had significantly worse mean scores for all the SF36 scales, except for body pain. Factors impacting HROOL of the patients were age, obesity, diabetes mellitus, and stone characteristics such as localisation (in the kidney) and recurrence.

Conclusions Post-lithotripsy, patients have a favourable HROOL compared with healthy volunteers. Further prospective studies are warranted to confirm these results owing to the inherent limitations of the cross-sectional design. 